Marquette University

e-Publications@Marquette

$1-1-2015$

\title{
Conditioned Pain Modulation Predicts Exercise- Induced Hypoalgesia in Healthy Adults
}

Kathy J. Lemley

Marquette University

Sandra K. Hunter

Marquette University, sandra.hunter@marquette.edu

Marie K. Hoeger Bement

Marquette University, mariehoeger.bement@marquette.edu

Accepted version. Medicine \& Science in Sports \& Exercise, Vol. 47, No. 1 (January 2015): 176-184.

DOI. (C) 2015 American College of Sports Medicine. Used with permission. 


\title{
Conditioned Pain Modulation Predicts Exercise-Induced Hypoalgesia in Healthy Adults
}

\author{
Kathy J. Lemley \\ Department of Physical Therapy, Marquette University, \\ Milwaukee, WI
}

Department of Physical Therapy, Concordia University Wisconsin, Mequon, WI

Sandra K. Hunter

Department of Physical Therapy, Marquette University, Milwaukee, WI

Marie K. Hoeger Bement

Department of Physical Therapy, Marquette University, Milwaukee, WI 
NOT THE PUBLISHED VERSION; this is the author's final, peer-reviewed manuscript. The published version may be accessed by following the link in the citation at the bottom of the page.

\section{Abstract}

Introduction: Conditioned pain modulation (CPM) is the concept that pain inhibits pain and has potential rehabilitation implications for exercise prescription. The purpose of this study was to determine whether changes in pressure pain perception after a thermal conditioning stimulus (i.e., CPM) was attenuated with aging and whether CPM predicted pain relief after exercise (exercise-induced hypoalgesia (EIH)) in healthy young and older adults.

Methods: Twenty young ( $21.9 \pm 3.3 \mathrm{yr}, 10 \mathrm{men})$ and 19 older $(72.0 \pm 4.5$ yr, 10 men) adults participated in three sessions: one familiarization and two experimental (EIH and CPM) sessions. Pressure pain perception was assessed using a weighted Lucite edge placed on the right index finger for 1 min. EIH was determined by measuring pressure pain perception before and after prolonged submaximal isometric contraction of the elbow flexors. CPM was assessed by measuring pressure pain perception at the finger while the foot was immersed in neutral water versus painful ice water.

Results: Young, but not older, adults reported a decrease in pressure pain at the finger while their foot was immersed in the ice water bath compared with the neutral bath (i.e., CPM, trial-age: $P=0.001$ ). Pressure pain ratings decreased after exercise $(P=0.03)$ that was perceived as painful (peak arm pain, $7.0 \pm 3.3$ ) for both young and older adults. Regression analysis showed that after controlling for age and baseline pain, CPM predicted EIH (model adjusted $\left.\mathrm{R}^{2}=0.23, \mathrm{P}=0.007\right)$.

Conclusions: CPM was attenuated in older adults, as measured with a noxious pressure stimulus after a thermal conditioning stimulus, and adults with greater CPM were more likely to report greater EIH.

Pain is the primary reason people seek health care and more than 100 million Americans experience chronic pain. ${ }^{12}$ In addition, the frequency of pain reports increases with age, with up to $80 \%$ of community-dwelling older adults experiencing some type of pain. $\underline{24}$ The effectiveness of exercise as a nonpharmacological means of pain management is well established. 8 Most types of exercise have been found to reduce pain sensitivity, a phenomenon known as exercise-



Previous research has demonstrated a reduction in pain at both the exercising limb $\frac{14,17,35}{}$ and distant sites, such as the nonexercising

Medicine \& Science in Sports \& Exercise, Vol 47, No. 1 (January 2015): pg. 176-184. DOI. This article is @ American College of Sports Medicine and permission has been granted for this version to appear in e-Publications@Marquette. American College of Sports Medicine does not grant permission for this article to be further copied/distributed or hosted elsewhere without the express permission from American College of Sports Medicine. 
limb. $9,16,17,21$ Attenuation of pain outside of the exercising muscles suggests that central or systemic mechanisms are involved in EIH. Both opioid and nonopioid mechanisms have been implicated ${ }^{3,8,13}$ and include the following: increase in beta endorphins, altered psychological states, interaction between the cardiovascular and pain processing systems, recruitment of high threshold motor units, and activation of the primary motor cortex. $\frac{8,9,15,27}{27}$

Conditioned pain modulation (CPM) is another potential factor that could influence EIH, especially given that exercise is sometimes perceived to be painful. With CPM, pain from a noxious stimulus (conditioning stimulus) results in the inhibition of pain during the application of a second noxious stimulus (test stimulus) applied elsewhere (i.e., "pain inhibits pain"). The difference in pain experienced with the test stimulus applied with and without the conditioning stimulus is a measure of CPM. The noxious conditioning stimulus activates descending inhibitory pathways, resulting in inhibition of extra-segmental spinal and trigeminal wide dynamic range neurons, $\underline{20}$ thereby decreasing pain associated with the test stimulus. It has been postulated that pain experienced during exercise may act as a conditioning stimulus, resulting in EIH. ${ }^{3}$

CPM protocols are used to indirectly measure the efficiency of descending inhibitory pathways. $\frac{36}{}$ Despite the frequent use of CPM protocols to assess endogenous pain modulation, intervention studies using CPM are limited. Two such studies have reported an association of CPM with pain reduction using transcutaneous electrical nerve stimulation in individuals with fibromyalgia 4 and pulsed radio frequency current in individuals with cluster headaches. $\stackrel{2}{\text { Specific to }}$ exercise, there are likely multiple mechanisms responsible for $\mathrm{EIH}, 3,8,13$ and CPM could provide an additive effect as the exercise becomes painful. For example, pain is seen to increase steadily during prolonged submaximal isometric contractions. $\stackrel{?}{ }$ Because greater CPM is produced by a stronger conditioning stimulus, 39 CPM may explain why in young adults, the greatest pain relief with isometric exercise occurred after contractions held for long duration compared with shorter durations. ${ }^{9,25}$ Few EIH studies have assessed muscle pain during exercise, and to the best of our knowledge, no study has directly examined the predictive relation of CPM to EIH in young or older adults. 
Older adults typically have an attenuated CPM response compared with that of young adults when using a thermal $6,29,38$ or electrical $\underline{38}$ test stimulus. Age-related differences in pain perception (independent of CPM) seem to be dependent upon the noxious stimulus used. $\frac{5,19}{}$ Whether the attenuation in CPM that occurs with aging is specific to modality and occurs with a pressure test stimulus is not known. A generalized reduction in CPM in older adults may help explain the absence of task differences in EIH after isometric contractions of varying durations. $\underline{21}$

The purpose of this study was to determine whether there are age-related differences in CPM using a noxious pressure test stimulus and whether there was a predictive relation between CPM and EIH using an exercise protocol that is typically perceived as painful. We hypothesized that 1 ) older adults would exhibit an attenuated CPM response compared with that of young adults using a noxious pressure test stimulus and 2) CPM would predict EIH so that those with less CPM would have less EIH.

\section{MATERIALS AND METHODS}

\section{Participants}

Fifty men and women were recruited for this study. Exclusion criteria included presence of acute or chronic pain, current use of analgesics or psychotropic medications, a score of less than 25/30 on the Mini-Mental Status Examination, any risk factors that would preclude participation in the exercise session or immersion in an ice water bath, and inability to tolerate the ice water bath. Eleven participants were unable to complete testing for the following reasons: history of cardiovascular disease $(n=5)$, musculoskeletal injury $(\mathrm{n}=1)$, symptoms associated with a neurological condition ( $\mathrm{n}$ $=2)$, and inability to tolerate the ice water bath $(n=3)$. Thirty-nine participants (10 young men (22.6 $\pm 3.8 \mathrm{yr}$ ), 10 young women $(21.3$ $\pm 2.9 \mathrm{yr}), 10$ older men (71.4 $\pm 4.7 \mathrm{yr}$ ) and nine older women (72.7 $\pm 4.6 \mathrm{yr})$ ) completed the protocol and were included in the final analysis. 
NOT THE PUBLISHED VERSION; this is the author's final, peer-reviewed manuscript. The published version may be accessed by following the link in the citation at the bottom of the page.

\section{Experimental Protocol}

Participants completed three sessions: one familiarization and two experimental sessions. All sessions were separated by approximately $1 \mathrm{wk}$, and experimental sessions were counterbalanced across sex and age. The protocol was approved by the institutional review boards at Marquette University and Concordia University Wisconsin.

Pressure pain perception was measured in all three sessions with a custom-made pressure pain device (Romus Inc., Milwaukee, WI) used previously in the assessment of EIH in young and older adults. $\frac{9-11,21}{}$ This device consisted of a weighted Lucite edge $(8 \times 1.5$ $\mathrm{mm}$ ) equivalent to a $1.5 \mathrm{~kg}$ mass placed on the dorsum of the right index finger midway between the proximal and distal interphalangeal joints for $1 \mathrm{~min}$. During the 1-min test, the subject was asked to say the word "pain" when the pressure changed to pain (i.e., pain threshold measured in seconds) and to rate the intensity of pain every $10 \mathrm{~s}$ using the $0-10$ numerical rating scale (NRS). ${ }^{22}$ The average of the six time points during the 1-min pressure pain test was used to identify potential relations between variables and group differences. Participants were informed that they could stop at any time if they reached pain tolerance.

During the familiarization session, participants signed an informed consent and were familiarized to the experimental procedures (e.g., pressure pain device and ice water bath). Pressure pain perception was measured twice, with a 30-min quiet rest between the two tests. Previous pilot data in our laboratory indicated that 30 min was necessary between the two pressure pain tests because $15 \mathrm{~min}$ would not provide adequate recovery for the pilot

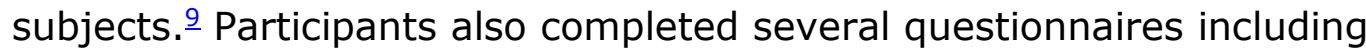
the Spielberger State-Trait Anxiety Inventory (STAI), , Pain Catastrophizing Scale (PCS), $\underline{33}$ Fear of Pain Questionnaire (FPQ), $\underline{23}$ Modified Pain Attitude Questionnaire-Revised (PAQ-R), ${ }^{40}$ and a selfreport measure of physical activity. $\underline{18}$ The state portion of the STAI was administered three times, before the initial pain test and again immediately after each of the two pressure pain tests. Because performance of maximal voluntary contractions (MVC) has been found to influence pain reports in young, ${ }^{9,14}$ and older 21 adults, determination of left elbow flexor MVC force was performed during the 
familiarization session after completion of the two pressure pain tests. A series of three MVC was performed with a 1-min rest between contractions. Participants were verbally encouraged to achieve maximal force on each attempt. The highest value of the three maximal efforts was used for calculation of the submaximal target force in the exercise session.

\section{Experimental Sessions}

\section{EIH session}

Pressure pain perception (i.e., pain threshold and pain ratings) was assessed before and after painful isometric contraction of the left elbow flexor muscles. Pressure pain perception was assessed within $60 \mathrm{~s}$ after the termination of the isometric contraction. A 30-min quiet rest period separated the completion of the first pain test and initiation of isometric exercise. The state portion of the STAI was administered before the initial pain test and again immediately after each of the two pressure pain tests (Fig. 1).

The isometric contraction was submaximal (25\% MVC) and sustained until task failure. Measurement of force was performed using established procedures. $\frac{9,21}{}$ Participants were seated upright in an adjustable chair with a padded nylon strap placed vertically over each shoulder to stabilize the subject and minimize shoulder region substitution. The left shoulder was placed in a position of slight abduction, with the elbow flexed to $90^{\circ}$ and resting on a padded support. The forearm was parallel to the floor in a neutral position midway between pronation and supination. The forearm, wrist, and hand were placed in a modified wrist-hand-thumb orthosis (Orthomerica, Newport Beach, CA), which was held in place by Velcro straps and rigidly attached to a force transducer (JR-3 Force-Moment Sensor; JR-3 Inc., Woodland, CA) mounted on a custom-designed adjustable support. The transducer measured the vertically directed force at the level of the wrist, which was recorded online using a Power 1401 A-D converter and Spike2 software (Cambridge Electronics Design, Cambridge, United Kingdom). The force signal was digitized at 500 samples per second.

During performance of the submaximal exercise task, participants were required to match the target force as displayed on a 
monitor. Participants were asked to rate the intensity of pain in the exercising elbow flexor muscles every 30 s throughout the duration of the exercise task. Participants were verbally encouraged to sustain the force for as long as possible. Task failure was determined using a computer-based software program that signaled when force output reached preestablished criterion of a decline of greater than $10 \%$ of the target value for three of five consecutive seconds. $\frac{9,21}{}$

\section{CPM session}

Pressure pain perception at the finger (test stimulus) was measured initially while the foot was immersed in a neutral $\left(25^{\circ} \mathrm{C} \pm\right.$ $1^{\circ} \mathrm{C}$ ) water bath and then $30 \mathrm{~min}$ later while the foot was immersed in a noxious ice $\left(2^{\circ} \mathrm{C} \pm 1^{\circ} \mathrm{C}\right)$ water bath (conditioning stimulus). ${ }^{4}$ The neutral water bath was used to control for potential distraction associated with water immersion. The difference in pressure pain ratings between the neutral bath and noxious water bath was used as a measure of CPM. Twenty seconds after foot immersion, participants reported pain intensity of the water bath using an 11-point (0-10) NRS and the 1-min pressure pain test was initiated (total duration of water bath immersion was approximately $80 \mathrm{~s}$ ). The NRS anchors were $0=$ no pain, $5=$ moderate pain, and $10=$ worst pain. $\underline{22}$ At completion of the pressure pain test and immediately before removing the foot from the water, participants again reported pain intensity for the water bath. The state portion of the STAI was administered before the initial pain test and again immediately after each of the two pressure pain tests (Fig. 1).

\section{Psychosocial Questionnaires}

\section{STAI}

The STAI 30 is an assessment of transient (state) and enduring (trait) levels of anxiety. Each subscale consists of 20 questions. Higher values are associated with greater levels of anxiety.

\section{PCS}

The $\mathrm{PCS}^{33}$ is a 13 -statement questionnaire that measures the degree to which an individual experiences exaggerated negative painrelated thoughts when anticipating or experiencing pain. Higher PCS total scores are indicative of greater catastrophizing. Participants 
NOT THE PUBLISHED VERSION; this is the author's final, peer-reviewed manuscript. The published version may be accessed by following the link in the citation at the bottom of the page.

were instructed to respond to the statements relative to their thoughts and feelings in general when in pain (i.e., dispositional).

FPQ-9

This nine question version of the FPQ has a scoring range of 945. Higher scores indicate greater fear of pain associated with specific situations. $\underline{23}$

\section{Modified PAQ-R}

The modified version of the PAQ- $R$ is a 23-item scale used for the assessment of stoicism and cautiousness about pain, with each dimension further subdivided into two subcategories. $\underline{40}$ Scale items are rated from 1 (strongly disagree) to 5 (strongly agree). Higher scores are associated with greater stoicism or cautiousness regarding pain.

\section{Physical Activity}

Participants self-reported physical activities in which they regularly participated (i.e., at least 10 times) over the past 12 months with a modified physical activity questionnaire $\frac{18}{}$ and reported as kilocalorie expenditure of energy per week.

\section{Statistical Analysis}

Data were analyzed using the Statistical Package for the Social Sciences (SPSS version 20; IBM, Chicago, IL) and were screened for outliers ( $>3$ SD from the mean) and missing data points. Outliers were altered to one-unit greater than the next extreme score. $\frac{34}{4}$ Missing pain ratings because of participants asking to stop the pressure pain test before the 60 -s duration were imputed with a rating of 10 .

There were some missing data points for pain threshold, with the greatest number of missing values in the CPM session. Failure to report pain threshold occurred 1.5 times more frequently during the ice water bath than during the neutral water bath (15 vs nine). More than one-half of the older adults (10/19), including seven of nine older women, did not say "pain" during the ice water bath, whereas one-quarter of the young adults (5/20) failed to do so. Because of the number of missing data points, pain threshold was excluded from further analysis. 
NOT THE PUBLISHED VERSION; this is the author's final, peer-reviewed manuscript. The published version may be accessed by following the link in the citation at the bottom of the page.

Separate mixed-design multivariate repeated-measures ANOVA with age group and sex as between-subject factors were used to assess change in pressure pain ratings for each session (trial (before vs after)-time (pressure pain rating every $10 \mathrm{~s}$ during the 60 -s pressure pain test)). The two levels of trial varied by session: pain ratings during the familiarization were assessed before and after quiet rest; CPM pain ratings were assessed with foot immersion during neutral water and ice water; and $\mathrm{EIH}$ pain ratings were assessed before and after exercise.

To determine whether anxiety was altered with the pain testing or intervention procedures, change in state anxiety was assessed for each session with separate mixed-design repeated-measures ANOVA (time [baseline, post-pressure pain test 1 , post-pressure pain test 2]). Age group and sex were between-subject factors. When a significant effect was found, simple contrasts followed by Bonferronicorrected t-tests for post hoc multiple comparisons were used to identify differences.

Multivariate repeated-measures ANOVA was used to assess for learning or order effects with the pressure pain device across sessions. The first pain assessment of each session (baseline measures) was compared for differences in pain ratings. Factors were session number (first, second, third) and time (pain ratings every 10 $\mathrm{s}$ during the 60-s pressure pain test). Paired t-test assessed for differences in baseline state anxiety between the two experimental sessions. Independent t-tests assessed for age and sex differences in dependent variables.

Pain ratings were averaged over the 60-s pressure pain test to determine change in pain with exercise (EIH) and ice water immersion (CPM). Change in pain ratings due to exercise or CPM was calculated by subtracting the average pain rating of the second pressure pain test from the average pain rating of the first pressure pain test (i.e., preexercise average - postexercise average or neutral water average - ice water average, respectively). To avoid the possible confounding effect of baseline pain, associations between variables were assessed using partial Pearson correlations with session baseline pain as a covariate. Session baseline pain is defined as the average pain rating of the initial pressure pain test for that session. Hierarchical regression analysis assessed for a predictive relation between CPM 
and EIH. Because baseline pain and age have been found to influence $\mathrm{EIH}, \underline{11,21}$ these potential confounders were controlled for in our regression analysis. Age group and preexercise average pressure pain ratings (i.e., EIH baseline pain) were entered (step 1) before the inclusion of CPM (step 2) in the prediction of EIH. A P value of $<0.05$ was used for statistical significance. Data are reported as mean \pm SD within the text and mean \pm SEM in the figures.

\section{Results}

Five outliers were identified and altered: one pain catastrophizing score, one baseline state anxiety score in each of the three sessions (total of three), and one physical activity score. No pain scores were $>3$ SD from the mean. Six participants (four older women, one older man, and one young woman) asked to stop at least once over the six pressure pain trials (two trials per session). Thus, a total of 28 missing pain ratings ( $2 \%$ ) for the three sessions were imputed with a rating of 10 .

\section{Familiarization Session}

A summary of participant characteristics (e.g., pain catastrophizing, physical activity) is shown in Table 1 . Pain ratings during the 1-min pressure pain test were similar before and after the 30-min quiet interval (trial, $\mathrm{P}>0.05$ ). In addition, there was no effect of session number on baseline pain $(P>0.05)$. Pain ratings increased during the 1-min pain test (time: $P<0.001$, [eta $]_{p}{ }^{2}=$ 0.85 ), with no difference between trials (trial-time, $P>0.05$ ). No age-related main or interaction effects were identified. Women reported higher pain ratings $\left(P=0.008,[\text { eta }]_{p}{ }^{2}=0.19\right)$ and a greater increase in pressure pain ratings than those reported by men during the 1-min pressure pain test (time-sex: $\mathrm{P}=0.017$, $[\text { eta }]_{\mathrm{p}}{ }^{2}=$ $0.35)$. State anxiety was higher before the first pain test ( $27.6 \pm 6.9)$ than that at the two post-pain test administrations (25.6 \pm 5.3 and $24.8 \pm 4.6$, respectively), with no difference between the latter two (time: $\mathrm{P}=0.01,[\mathrm{eta}]_{\mathrm{p}}{ }^{2}=0.24$ ). There were no main or interaction effects for either age or sex in state anxiety.

\section{EIH Session: Pain Ratings}

Average pressure pain ratings decreased approximately $14 \%$ after exercise $\left(P=0.03,[\text { eta }]_{p}^{2}=0.12\right)$ (Table 1 and Fig. $\left.2 A\right)$. There was a significant main effect for age $\left.(P=0.02 \text {, [eta }]_{p}^{2}=0.15\right)$; older 
adults reported higher pressure pain ratings than those reported by young adults. No significant interaction effects were identified for age; young and older adults had similar decreases in pressure pain reports after exercise (trial-age, $\mathrm{P}>0.05$ ).

A significant main effect for sex was also identified. Women reported higher pressure pain ratings than those reported by men ( $P$ $\left.=0.001,[\text { eta }]_{p}^{2}=0.30\right)$. Women also reported a steeper increase in pressure pain ratings than that reported by men during the 1-min pressure pain test (time-sex: $P=0.024$, $[\text { eta }]_{p}{ }^{2}=0.33$ ). Both men and women experienced a similar magnitude of EIH (trial-sex: P > 0.05) (Fig. 2B).

No group differences were identified for the perception of exercise-induced arm pain. Young and older adults perceived exercise as equally painful as did men and women $(P>0.05)$ (Table 1$)$. Peak arm pain during exercise was not associated with EIH $(P>0.05)$.

\section{CPM Session: Pain Ratings}

Pressure pain ratings (measured at the finger) decreased approximately $25 \%$ while the foot was immersed in the ice water bath compared with the neutral water bath $\left(P<0.001\right.$, $[\text { eta }]_{p}{ }^{2}=$ 0.38 ) (Table 1 ). The rise in pressure pain ratings during the 1 -min pressure pain test was also less when the foot was immersed in the ice water bath (trial-time: $P<0.001,[\text { eta }]_{p}^{2}=0.55$ ). There was a significant main effect for age $\left(P=0.006\right.$, $\left.[\text { eta }]_{p}{ }^{2}=0.20\right)$ and a trial and age interaction $\left(P=0.001,[\text { eta }]_{p}{ }^{2}=0.29\right)$. Post hoc analysis revealed a reduction of pressure pain with the foot immersed in the ice water bath compared with the neutral water bath only for the young adults and not the older adults (young: $P<0.001$, Cohen $d=$ 1.08; older: $P>0.05$, Cohen $d=0.05)$ (Fig. 3A).

Women reported higher pressure pain ratings at the finger than those reported by men $\left(P=0.001\right.$, $\left.[\text { eta }]_{p}{ }^{2}=0.26\right)$, with a greater increase in pain ratings during the 1 -min pressure pain test (time-

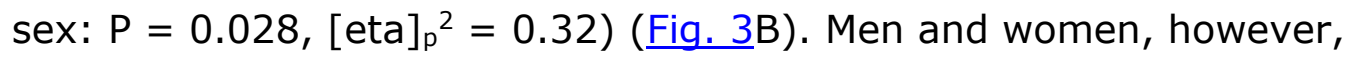
reported similar pressure pain reductions with foot immersion in the ice water bath compared with foot immersion in the neutral water bath (trial-sex, $P>0.05)$. An age and sex interaction $(P=0.039$, $[\text { eta }]_{\mathrm{p}}{ }^{2}=0.12$ ) was identified with older women reporting higher pressure pain ratings than those reported by younger women in both 
water baths (neutral water: $5.91 \pm 2.21$ vs $3.57 \pm 1.69, P=0.018$, Cohen $d=1.19$; ice water: $5.50 \pm 2.44$ vs $1.92 \pm 1.50, P=0.001$, Cohen $d=1.77)$. No age-related difference in pressure pain ratings was found for men $(P>0.05)$.

Average foot pain intensity during immersion in the ice water bath was $4.2 \pm 2.5$ at $20 \mathrm{~s}$ immersion (immediately before the onset of the test stimulus) and $5.7 \pm 2.6$ immediately before removing the foot from the ice water bath (Table 1). There were no age or sex differences in the perception of pain for the water baths at either time point $(P>0.05)$. Perception of pain intensity for the ice water bath was not associated with magnitude of CPM $(P>0.05)$.

\section{Strength and Time to Task Failure}

Strength (MVC force) did not differ between young and older adults ( $225 \pm 78$ vs $210 \pm 82 \mathrm{~N}, \mathrm{P}>0.05$, Cohen $\mathrm{d}=0.19)$. Time to task failure for the submaximal isometric contraction was also similar for young and older adults (587 \pm 235 vs $739 \pm 379$ s, P > 0.05, Cohen $d=0.48)$. Time to task failure did not differ between men and women (601 \pm 337 vs $725 \pm 294$ s, $P>0.05$, Cohen $d=0.66)$. Men, however, were stronger than women (282 \pm 48 vs $150 \pm 38 \mathrm{~N}, \mathrm{P}<$ 0.001 , Cohen $d=3.0)$, and MVC force was negatively associated with time to task failure $(r=-0.47, P=0.003)$, such that stronger individuals had a briefer time to failure. Strength was also negatively associated with average pain ratings before $(r=-0.52, P=0.001)$ and after $(r=-0.53, P=0.001)$ the submaximal isometric fatiguing contraction, but neither strength nor time to task failure was associated with change in pain after exercise $(P>0.05)$.

\section{Psychosocial Variables}

\section{Anxiety}

Initial state anxiety was similar between experimental sessions $(P>0.05)$. There was no change in anxiety over time in the CPM session $(P>0.05)$. During the exercise session, state anxiety was similar before and after the first pressure pain test but increased after exercise (time: $P=0.005$, $[\text { eta }]_{p}^{2}=0.27$ ). Older adults were less anxious than young adults in both sessions (exercise age: $\mathrm{P}=0.047$, $[\text { eta }]_{p}^{2}=0.11$; CPM age: $P=0.030$, $\left.[\text { eta }]_{p}^{2}=0.13\right)$ (Table 1$)$. There were no significant main or interaction effects for sex in either session. 
NOT THE PUBLISHED VERSION; this is the author's final, peer-reviewed manuscript. The published version may be accessed by following the link in the citation at the bottom of the page.

As state anxiety was stable over time in the CPM session, the three scores were averaged and the average score was used for correlational analyses. State anxiety was positively associated with change in pain ratings for the CPM session ( $r=0.423, P=0.008)$; those participants with higher state anxiety experienced greater reductions in pain with the ice water bath. No association of state anxiety with $\mathrm{EIH}$ was found, nor was trait anxiety related to change in pain in either session $(P>0.05)$.

Pain catastrophizing, fear of pain, pain attitude, and physical activity

No age or sex differences were identified for pain catastrophizing, fear of pain, physical activity, or pain attitude. In addition, no relations were identified for pain catastrophizing, fear of pain, or pain attitude, with change in pain in either the CPM or EIH session. Physical activity was moderately related to change in pain ratings with CPM $(r=0.368, P=0.023)$ but not after exercise $(P>$ 0.05). More physically active participants experienced greater CPM.

\section{Hierarchical Regression Analysis}

Baseline pain in the CPM session (i.e., average pressure pain ratings with the foot immersed in the neutral water bath) showed a strong positive relation with baseline pain in the EIH session (i.e., preexercise average pressure pain ratings: $r=0.79, \mathrm{P}<0.001$ ). After controlling for age group and $\mathrm{EIH}$ session baseline pain, the change in pain with CPM uniquely explained $8.8 \%$ of the variance in change in pain ratings after exercise. Individuals with greater reductions in pressure pain in the ice water bath also experienced greater reductions in pain after exercise (Fig. 4). Of the three predictor variables, only CPM and baseline pain uniquely predicted $\mathrm{EIH}$. As a whole, the final model explained $23 \%$ of the variance in $\mathrm{EIH}\left(\mathrm{F}_{3,35}=4.71, \mathrm{P}=0.007\right)$ (Table 2).

\section{Discussion}

It has been theorized that painful exercise may activate CPM, thereby explaining the systemic reduction in pain sensitivity often seen after exercise. We examined the effect of age on CPM using a pressure pain test stimulus and the predictive relation of CPM for $\mathrm{EIH}$ after a low-intensity prolonged isometric contraction. The main findings of this study were as follows: 1) CPM was attenuated in older

Medicine \& Science in Sports \& Exercise, Vol 47, No. 1 (January 2015): pg. 176-184. DOI. This article is (C) American College of Sports Medicine and permission has been granted for this version to appear in e-Publications@Marquette. American College of Sports Medicine does not grant permission for this article to be further copied/distributed or hosted elsewhere without the express permission from American College of Sports Medicine. 
adults when using a noxious pressure test stimulus and 2) the reduction in pain ratings after isometric exercise was predicted by the CPM response. To our knowledge, this is the first study to demonstrate that CPM is predictive of the pain response after exercise.

An attenuated CPM response in older adults using electrical and thermal test stimuli has been shown previously. $\frac{6,29,38}{}$ The present study extends these results to a tonic pressure test stimulus. Only young adults experienced CPM, whereas older adults experienced a broad range in their pain response between individuals, as follows: nine older adults reported no change in pain, five reported hyperalgesia, and five reported hypoalgesia. This finding has important clinical implications when using interventions mediated by activation of descending inhibitory pathways. Older adults, as a whole, may have a lower response compared with that of younger adults to such interventions, but some older adults will likely find these interventions as effective as their younger counterparts in the management of pain. One limitation of this study was that the small sample size precluded identification of characteristics of those older adults experiencing hypoalgesia as opposed to hyperalgesia with CPM testing.

Our findings showed that CPM predicted the EIH response in young and older adults, such that those individuals that demonstrated a greater ability to activate descending inhibitory pathways reported greater $\mathrm{EIH}$. Although this association is not causal, these results provide insight into the variability in the pain response that is often reported after exercise. 11,25 For example, others have suggested that the attenuated pain relief or even pain exacerbation after exercise in some patients with pain may be due to abnormal descending

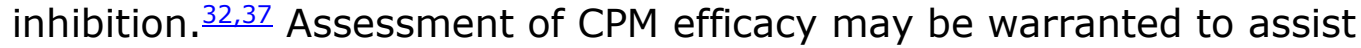
in clinical decision making to individualize pain management interventions and establish the characteristics of those who will likely benefit most.

Pain relief occurs with nonpainful and painful isometric contractions (e.g., short-duration low-intensity contractions or brief MVC). .21 When exercise is painful, however, CPM may work as an additive effect for pain relief. Other studies have demonstrated the additive effect of CPM with transcranial stimulation of the primary 
motor cortex in elevating pressure pain thresholds. $\stackrel{28}{\text { Specific to }}$ exercise, the ability to engage descending inhibitory pathways may augment another unknown mechanism to explain the effect of exercise duration on $\mathrm{EIH}$ in young adults. $\frac{9,25}{}$ Isometric contractions of both short and longer duration produce $\mathrm{EIH}$; however, the greatest $\mathrm{EIH}$ response occurs with longer-duration isometric contractions that typically induce more pain as the duration increases. ${ }^{\underline{7}}$ Involvement of CPM in EIH may also help explain the lower effect size for EIH in older adults compared with that in young adults using the same exercise protocol in an earlier study $\underline{21}$ because older adults have reduced CPM. $\underline{6,29,38}$

Despite CPM predicting EIH, the relation between CPM and EIH was not strong, indicating that there are other factors involved in pain reduction after exercise. We investigated several potential contributors to the EIH response. Baseline pain perception during the exercise session partially explained the variance in $\mathrm{EIH}$ and had a similar beta value as CPM; individuals with higher preexercise pain ratings experienced greater reductions in pain after exercise. This is consistent with our previous finding in individuals with fibromyalgia, where participants with lower preexercise pain thresholds were more likely to experience reductions in pain after isometric exercise. $\frac{11}{}$ In the fibromyalgia study, we hypothesized that greater experimental pain sensitivity may also result in greater pain during the exercise task, thereby producing greater EIH. Pain during exercise was not assessed in the fibromyalgia study to confirm this relation; however, no relation was found between peak pain during exercise and either baseline pain sensitivity or EIH in the current study.

A variety of psychosocial factors have been shown to be associated with pain perception $1,27,33,40$ and have the potential to moderate EIH. To assess for possible contributions of psychosocial factors to EIH, we examined anxiety, pain catastrophizing, fear of pain, and pain attitude. Despite a small increase in state anxiety after exercise, neither trait nor state anxiety correlated with $\mathrm{EIH}$, which is consistent with our previous findings in both young and older adults. $\frac{9,21}{}$ A positive association with state anxiety was found for CPM, although this may have been mediated by age. Young adults had slightly higher anxiety scores than older adults, a finding previously 
reported in the literature. $\underline{\underline{31}}$ We found neither pain catastrophizing, fear of pain, nor pain attitude to be related to either EIH or CPM.

In addition, we examined the potential contribution of physical activity to the pain response to exercise and CPM. Interestingly, more physically active participants experienced greater CPM. This finding is consistent with a recent report of a predictive relation of total and vigorous physical activity and CPM. $\stackrel{26}{ }$ Participation in regular physical activity may affect a person's ability to activate descending inhibitory pathways, or an alternative explanation is that individuals with more efficient activation of inhibitory pathways may find intense exercise to be less unpleasant and thus participate more in physical activity. The former has potential clinical implications for rehabilitation, in that CPM may be modified with physical activity whereas the latter has implications for health promotion and wellness. Whether physical activity can be manipulated to alter CPM or minimize its attenuation with aging is not known. Furthermore, all our participants were healthy, and most of them participated in regular physical activity, which may not be representative of the general or clinical pain populations.

Considering that CPM was a predictor of EIH and older adults experienced less CPM than young adults, an age-related difference in EIH magnitude might be anticipated. Our results did not show a statistical difference between age groups under the current experimental protocol. Previous work in our laboratory, however, has shown a moderate EIH effect size for young adults ${ }^{9}$ and a small effect size for older adults. $\underline{21}$ In the current study, the effect size for both young and older adults was substantially reduced compared with our earlier findings. The main difference between the earlier protocols and the current protocol are the parameters of the pressure pain device. Earlier studies used a $1.0-\mathrm{kg}$ mass and 2-min test duration; the current study used a $1.5-\mathrm{kg}$ mass for $1 \mathrm{~min}$, prompted by the need to limit the duration of the ice water bath. Comparison of pain reports for the two protocols showed that peak pain ratings and average pain ratings were similar. Thus, although participants perceived similar pain intensity with the two protocols, the rate of rise to peak pain was significantly more rapid with the heavier mass. The reduced effect size with change in stimulus parameters therefore suggests that magnitude of EIH may be dependent on the rate at which pain

Medicine \& Science in Sports \& Exercise, Vol 47, No. 1 (January 2015): pg. 176-184. DOI. This article is ( American College of Sports Medicine and permission has been granted for this version to appear in e-Publications@Marquette. American College of Sports Medicine does not grant permission for this article to be further copied/distributed or hosted elsewhere without the express permission from American College of Sports Medicine. 
intensity increases during a noxious stimulus. Future experiments should be conducted using a lighter weight to avoid pain saturation to more clearly identify whether older adults have attenuated EIH after painful isometric exercise.

\section{Conclusions}

Older adults had an attenuated CPM response compared with that of young adults using a tonic pressure test stimulus, and this CPM response was predictive of EIH in both older and younger adults. Understanding the relation between CPM and EIH could help establish the principles necessary to create clinical practice paradigms for the use of exercise as an effective pain management tool while also establishing profiles of people who will benefit most.

This work was partly supported by a Concordia University Wisconsin Intramural research grant to K. J. L.

No conflicts of interest are declared by any of the authors.

The results of this study do not constitute endorsement by the American College of Sports Medicine.

\section{References}

${ }^{1}$ Campbell CM, Kronfli T, Buenaver LF, et al. Situational versus dispositional measurement of catastrophizing: associations with pain responses in multiple samples. J Pain. 2010; 11 (5): 443-53.e2.

${ }^{2}$ Chua $\mathrm{NH}$, Vissers KC, Wilder-Smith $\mathrm{OH}$. Quantitative sensory testing may predict response to sphenopalatine ganglion pulsed radiofrequency treatment in cluster headaches: a case series. Pain Pract. 2011; 11 (5): 439-45.

${ }^{3}$ Cook DB, Koltyn KF. Pain and exercise. Int J Sport Psychol. 2000; 31: 25677.

${ }^{4}$ Dailey DL, Rakel BA, Vance CG, et al. Transcutaneous electrical nerve stimulation reduces pain, fatigue and hyperalgesia while restoring central inhibition in primary fibromyalgia. Pain. 2013; 154 (11): 2554-62.

${ }^{5}$ Edwards RR, Fillingim RB. Age-associated differences in responses to noxious stimuli. J Gerontol A Biol Sci Med Sci. 2001; 56 (3): M180-5.

${ }^{6}$ Edwards RR, Fillingim RB, Ness TJ. Age-related differences in endogenous pain modulation: a comparison of diffuse noxious inhibitory controls in healthy older and younger adults. Pain. 2003; 101 (1-2): 155-65.

Medicine \& Science in Sports \& Exercise, Vol 47, No. 1 (January 2015): pg. 176-184. DOI. This article is ( American College of Sports Medicine and permission has been granted for this version to appear in e-Publications@Marquette. American College of Sports Medicine does not grant permission for this article to be further copied/distributed or hosted elsewhere without the express permission from American College of Sports Medicine. 
NOT THE PUBLISHED VERSION; this is the author's final, peer-reviewed manuscript. The published version may be accessed by following the link in the citation at the bottom of the page.

7 Frey Law LA, Lee JE, McMullen TR, Xia T. Relationships between maximum holding time and ratings of pain and exertion differ for static and dynamic tasks. Appl Ergon. 2010; 42 (1): 9-15.

${ }^{8}$ Hoeger Bement MK. Exercise-induced hypoalgesia: an evidenced-based review. In: Sluka KA, editor. Management and Mechanisms of Pain for the Physical Therapist. Seattle: IASP Press; 2009. pp. 143-66.

${ }^{9}$ Hoeger Bement MK, Dicapo J, Rasiarmos R, Hunter SK. Dose response of isometric contractions on pain perception in healthy adults. Med Sci Sports Exerc. 2008; 40 (11): 1880-9.

10 Hoeger Bement MK, Rasiarmos RL, DiCapo JM, et al. The role of the menstrual cycle phase in pain perception before and after an isometric fatiguing contraction. Eur J Appl Physiol. 2009; 106 (1): 105-12.

${ }^{11}$ Hoeger Bement MK, Weyer A, Hartley S, Drewek B, Harkins AL, Hunter SK. Pain perception after isometric exercise in women with fibromyalgia. Arch Phys Med Rehabil. 2011; 92 (1): 89-95.

12 Institute of Medicine: Committee on Advancing Pain Research Care and Education. Relieving Pain in America: A Blueprint for Transforming Prevention, Care, Education, and Research. Washington (DC): The National Academies Press; 2011. 382 p. Available from: http://www.nap.edu/catalog.php?record id $=13172$.

${ }^{13}$ Koltyn KF. Analgesia following exercise: a review. Sports Med. 2000; 29 (2): 85-98.

${ }^{14}$ Koltyn KF, Trine MR, Stegner AJ, Tobar DA. Effect of isometric exercise on pain perception and blood pressure in men and women. Med $\mathrm{SCi}$ Sports Exerc. 2001; 33 (2): 282-90.

${ }^{15}$ Koltyn KF, Umeda M. Exercise, hypoalgesia and blood pressure. Sports Med. 2006; 36 (3): 207-14

${ }^{16}$ Koltyn KF, Umeda M. Contralateral attenuation of pain after short-duration submaximal isometric exercise. J Pain. 2007; 8 (11): 887-92

17 Kosek E, Lundberg L. Segmental and plurisegmental modulation of pressure pain thresholds during static muscle contractions in healthy individuals. Eur J Pain. 2003; 7 (3): 251-8.

18 Kriska AM, Bennett PH. An epidemiological perspective of the relationship between physical activity and NIDDM: from activity assessment to intervention. Diabetes Metab Rev. 1992; 8 (4): 355-72.

19 Lautenbacher S. Experimental approaches in the study of pain in the elderly. Pain Med. 2012; 13 (2 Suppl): S44-50.

Medicine \& Science in Sports \& Exercise, Vol 47, No. 1 (January 2015): pg. 176-184. DOI. This article is (c) American College of Sports Medicine and permission has been granted for this version to appear in e-Publications@Marquette. American College of Sports Medicine does not grant permission for this article to be further copied/distributed or hosted elsewhere without the express permission from American College of Sports Medicine. 
NOT THE PUBLISHED VERSION; this is the author's final, peer-reviewed manuscript. The published version may be accessed by following the link in the citation at the bottom of the page.

20 Le Bars D, Willer JC. Diffuse noxious inhibitory controls (DNIC). In: Basbaum AI, Bushnell MC, editors. Science of Pain. Oxford (UK): Elsevier; 2009. pp. 763-73.

${ }^{21}$ Lemley KJ, Drewek B, Hunter SK, Hoeger Bement MK. Pain relief after isometric exercise is not task-dependent in older men and women. Med Sci Sports Exerc. 2014; 46 (1): 185-91

22 McCaffery M, Pasero C. Assessment: underlying complexities, misconceptions, and practical tools. In: Bowlus B, editor. Pain: Clinical Manual. St. Louis (MO): Mosby; 1999. pp. 35-102.

23 McNeil DW, Vowles KE. Assessment of fear and anxiety associated with pain: conceptualization, methods, and measures. In: Asmundson GJ, Vlaeyen JW, Crombez G, editors. Understanding and Treating Fear of Pain. New York (NY): Oxford University Press; 2004. pp. 189-211.

24 Mobily PR, Herr KA, Clark MK, Wallace RB. An epidemiologic analysis of pain in the elderly: the Iowa $65+$ rural health study. J Aging Health. $1994 ; 6$ (2): 139-54

25 Naugle KM, Fillingim RB, Riley JL 3rd. A meta-analytic review of the hypoalgesic effects of exercise. J Pain. 2012; 13 (12): 1139-50.

${ }^{26}$ Naugle KM, Riley JL 3rd. Self-reported physical activity predicts pain inhibitory and facilitatory function. Med Sci Sports Exerc. 2014; 46 (3): 622-9.

27 Okawa K, Ichinohe T, Kaneko Y. Anxiety may enhance pain during dental treatment. Bull Tokyo Dent Coll. 2005; 46 (3): 51-8.

${ }^{28}$ Reidler JS, Mendonca ME, Santana MB, et al. Effects of motor cortex modulation and descending inhibitory systems on pain thresholds in healthy subjects. J Pain. 2012; 13 (5): 450-8.

${ }^{29}$ Riley JL 3rd, King CD, Wong F, Fillingim RB, Mauderli AP. Lack of endogenous modulation and reduced decay of prolonged heat pain in older adults. Pain. 2010; 150 (1): 153-60.

30 Spielberger CD, Gorsuch RL, Lushene PR, Vagg PR, Jacobs AG. Manual for the State-Trait Anxiety Inventory (Form Y). Palo Alto (CA): Consulting Psychologists Press; 1983. p. 36.

${ }^{31}$ Stanley MA, Beck JG, Zebb BJ. Psychometric properties of four anxiety measures in older adults. Behav Res Ther. 1996; 34 (10): 827-38.

32 Staud R, Robinson ME, Price DD. Isometric exercise has opposite effects on central pain mechanisms in fibromyalgia patients compared to normal controls. Pain. 2005; 118 (1-2): 176-84.

33 Sullivan MJL, Bishop SR, Pivik J. The pain catastrophizing scale: development and validation. Psychol Assess. 1995; 7 (4): 524-32.

Medicine \& Science in Sports \& Exercise, Vol 47, No. 1 (January 2015): pg. 176-184. DOI. This article is (c) American College of Sports Medicine and permission has been granted for this version to appear in e-Publications@Marquette. American College of Sports Medicine does not grant permission for this article to be further copied/distributed or hosted elsewhere without the express permission from American College of Sports Medicine. 
NOT THE PUBLISHED VERSION; this is the author's final, peer-reviewed manuscript. The published version may be accessed by following the link in the citation at the bottom of the page.

34 Tabachnick BG, Fidell LS. Using Mulitvariate Statistics. 4th ed. Boston (MA): Allyn and Bacon; 2001. p. 966.

35 Umeda M, Newcomb LW, Ellingson LD, Koltyn KF. Examination of the dose-response relationship between pain perception and blood pressure elevations induced by isometric exercise in men and women. Biol Psychol. 2010; 85 (1): 90-6.

${ }^{36}$ van Wijk G, Veldhuijzen DS. Perspective on diffuse noxious inhibitory controls as a model of endogenous pain modulation in clinical pain syndromes. J Pain. 2010; 11 (5): 408-19.

37 Vierck CJ Jr, Staud R, Price DD, Cannon RL, Mauderli AP, Martin AD. The effect of maximal exercise on temporal summation of second pain (windup) in patients with fibromyalgia syndrome. J Pain. 2001; 2 (6): 334-44.

${ }^{38}$ Washington LL, Gibson SJ, Helme RD. Age-related differences in the endogenous analgesic response to repeated cold water immersion in human volunteers. Pain. 2000; 89 (1): 89-96.

39 Willer JC, De Broucker T, Le Bars D. Encoding of nociceptive thermal stimuli by diffuse noxious inhibitory controls in humans. $J$ Neurophysiol. 1989; 62 (5): 1028-38.

40 Yong HH, Bell R, Workman B, Gibson SJ. Psychometric properties of the Pain Attitudes Questionnaire (revised) in adult patients with chronic pain. Pain. 2003; 104 (3): 673-81.

Key Words: Pain; Aging; Sex Differences; Elbow Flexor Muscles

Medicine \& Science in Sports \& Exercise, Vol 47, No. 1 (January 2015): pg. 176-184. DOI. This article is (C) American College of Sports Medicine and permission has been granted for this version to appear in e-Publications@Marquette. American College of Sports Medicine does not grant permission for this article to be further copied/distributed or hosted elsewhere without the express permission from American College of Sports Medicine. 
NOT THE PUBLISHED VERSION; this is the author's final, peer-reviewed manuscript. The published version may be accessed by following the link in the citation at the bottom of the page.

\section{Image Gallery}

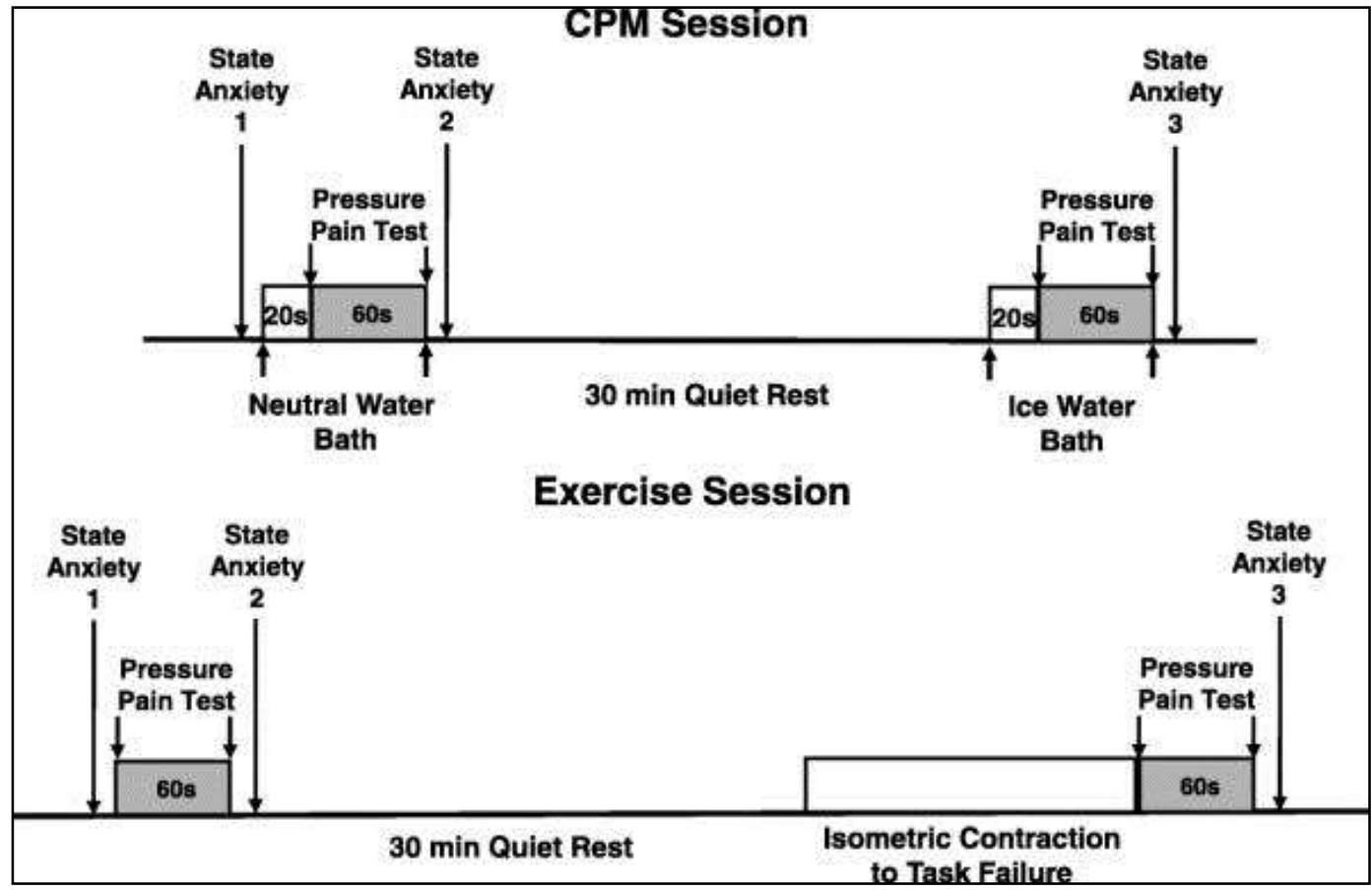

FIGURE 1. Study design. Sessions were counterbalanced across age and sex and were separated by approximately $1 \mathrm{wk}$. Note that this figure is not time to scale.

\begin{tabular}{|c|c|c|c|c|c|}
\hline & All Participants & Young, Adults & Oider Adults & Women & Men \\
\hline Pain cxtastrophizing & $10.8+8.0$ & $12.3+8.3$ & $9.2+7.5$ & $8.9+73$ & $12.6+8.3$ \\
\hline Fear of pain & $12.8+5.2$ & $12.7+4.7$ & $13.1+5.7$ & $13.4+6.0$ & $12.4+4.3$ \\
\hline Pain attitude-stoicism reficence & $3.4 \pm 0.5$ & $33 \pm 0.6$ & $3.4 \pm 0.4$ & $32 \pm 0.5$ & $3.5+0.6$ \\
\hline Pain attitude--stocism superiority & $3.3 \pm 0.7$ & $3.3+0.8$ & $3.4 \pm 0.5$ & $3.3+0.7$ & $3.3 \pm 0.7$ \\
\hline Pain attitude-cautiousness setf-doubt & $2.7+0.8$ & $28+0.8$ & $2.6+0.8$ & $26+08$ & $2.8+0.8$ \\
\hline Pain attitude-cautiousness reluctance & $3.6+0.6$ & $3.6+0.7$ & $3.6+0.5$ & $3.5+0.6$ & $3.6+0.7$ \\
\hline Trait anxisty & $30.8+7.8$ & $33.6 \pm 8.0$ & $28.0+6.8$ & $31.6 \pm 7.4$ & $30.2+8.4$ \\
\hline Physical activity & $42.8 \pm 32.5$ & $49.5+34.8$ & $35.8 \div 28.8$ & $39.6 \pm 40.9$ & $45.8+22.3$ \\
\hline \multicolumn{6}{|l|}{ Exercise session } \\
\hline State anxiety time 1 & $26.7+7.6$ & $28.1+7.7$ & $25.2+7.3$ & $26.2+7.4$ & $27.1+7.9$ \\
\hline Time 2 & $26.7+7.3$ & $28.6 \pm 7.6$ & $24.7+6.6$ & $26.2 \neq 7.1$ & $27.2+7.7$ \\
\hline Time 3 & $29.2 \times 7.9$ & $32.6+8.2$ & $25.7 \pm 5.8$ & $29.5 \div 8.1$ & $29.0 \pm 7.8$ \\
\hline Peak amn pain & $6.97+3.25$ & $6.63+3.20$ & $7.34+3.35$ & $7.00+3.32$ & $6.95+3.27$ \\
\hline Preexerclse average PPR & $3.42+2.41$ & $2.88+2.09$ & $4.00+2.64$ & $4.57+2.68$ & $2.33+1.50$ \\
\hline Postbotercise average PPR & $2.94+2.32$ & $2.15+1.85$ & $3.77+2.42$ & $4.06+2.35$ & $1.87+1.74$ \\
\hline EHH & $0.48 \pm 1.36$ & $0.73 \pm 1.38$ & $0.23 \div 1.33$ & $0.51 \pm 1.43$ & $0.46 \pm 1.34$ \\
\hline \multicolumn{6}{|l|}{ CPM session } \\
\hline State andies time 1 & $27.6+7.2$ & $29.7+7.5$ & $25.4 \pm 6.3$ & $26.5 \pm 6.5$ & $28.6+7.8$ \\
\hline Time 2 & $28.1+7.8$ & $30.9+7.4$ & $25.2+72$ & $27.1+7.4$ & $29.0+8.2$ \\
\hline Time 3 & $27.6 \pm 7.0$ & $29.8+7.0$ & $25.4 \div 6.3$ & $26.6 \pm 6.0$ & $28.6+7.8$ \\
\hline foot pain in ika water bath (20 s) & $4.23 \pm 2.50$ & $4.45 \pm 2.01$ & $4.00 \div 2.96$ & $4.63 \pm 2.61$ & $3.85+2.39$ \\
\hline foot pain in ice water bath (80 s) & $5.69+2.59$ & $5.85+2.08$ & $5.53+3.08$ & $6.47 \pm 2.20$ & $4.95+2.76$ \\
\hline Neutral water average PPG & $3.58+2.24$ & $3.15+1.62$ & $4.03+273$ & $4.68+2.25$ & $2.54+1.72$ \\
\hline loe water grerage PPR & $2.67 \pm 2.52$ & $1.52 \pm 1.40$ & $3.88 \div 2.89$ & $3.61 \pm 2.67$ & $1.77+2.06$ \\
\hline $\mathrm{com}$ & $0.91 \pm 1.40$ & $1.63 \pm 0.91$ & $0.15 \div 1.45$ & $1.06 \pm 1.54$ & $0.77 \pm 1.28$ \\
\hline
\end{tabular}

TABLE 1. Participant characteristics.

Medicine \& Science in Sports \& Exercise, Vol 47, No. 1 (January 2015): pg. 176-184. DOI. This article is (c) American College of Sports Medicine and permission has been granted for this version to appear in e-Publications@Marquette. American College of Sports Medicine does not grant permission for this article to be further copied/distributed or hosted elsewhere without the express permission from American College of Sports Medicine. 
NOT THE PUBLISHED VERSION; this is the author's final, peer-reviewed manuscript. The published version may be accessed by following the link in the citation at the bottom of the page.

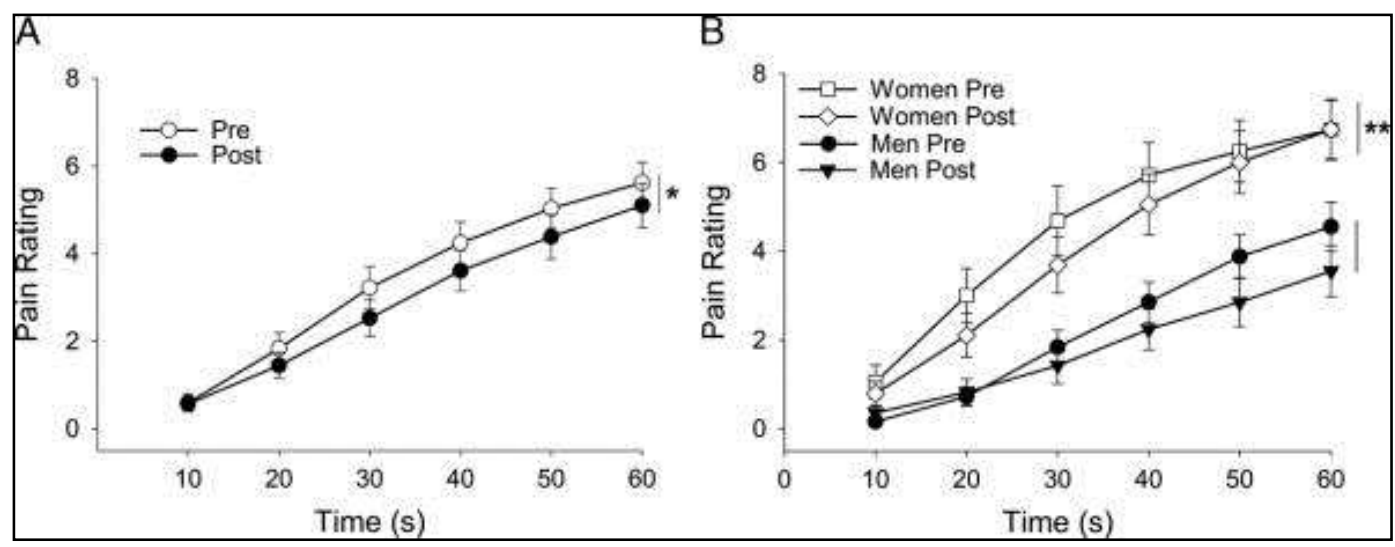

FIGURE 2. Exercise session pain ratings. Pressure pain ratings before (pre) and after (post) isometric exercise. Pressure pain ratings decreased after exercise (A). Women reported higher pain ratings than those reported by men during the 1-min pressure pain test, but men and women reported a similar decrease in pressure pain ratings after exercise (B). Data are represented as mean +/- SEM. *Trial, P P $<0.05$.

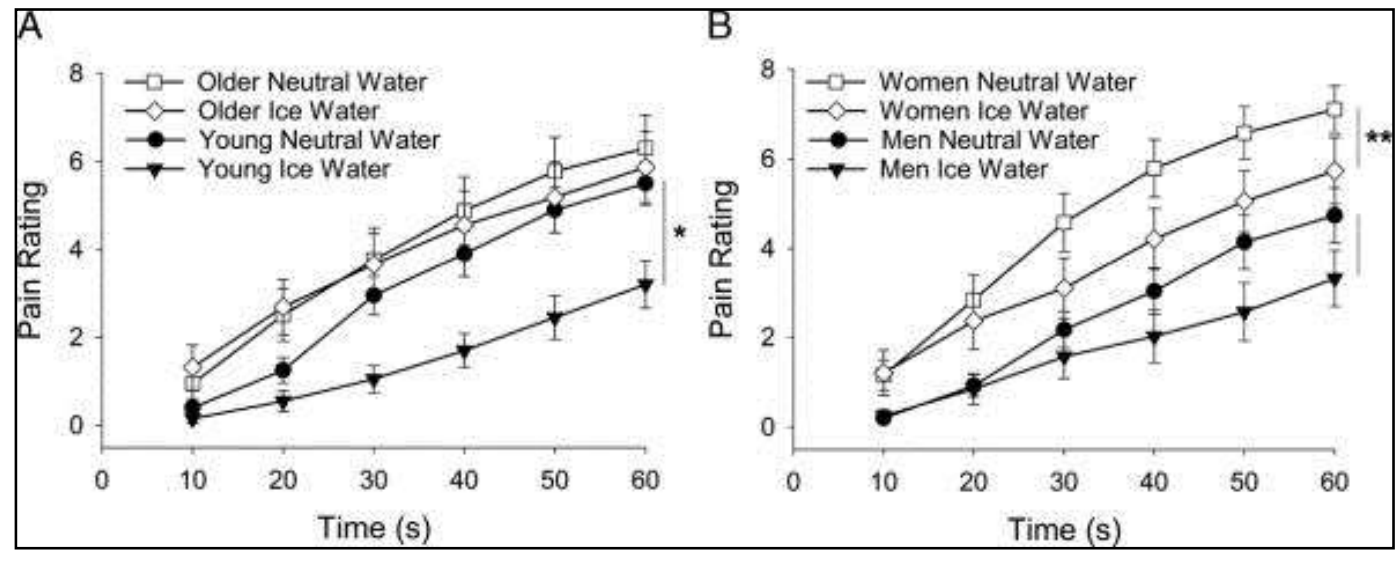

FIGURE 3. CPM session pain ratings. Age and sex differences in pressure pain ratings with the foot immersed in the neutral water bath compared with ratings with the ice water bath. Only the young adults reported a decrease in pressure pain ratings while their foot was immersed in the ice water bath compared with the neutral water bath (A). Women reported higher pressure pain ratings than those reported by men for both the neutral and ice water baths, but both men and women experienced similar reductions in pressure pain with the foot immersed in the ice water bath compared with the neutral water bath (B). Data are represented as mean +/- SEM. *Trial, P P = 0.001 . 
NOT THE PUBLISHED VERSION; this is the author's final, peer-reviewed manuscript. The published version may be accessed by following the link in the citation at the bottom of the page.

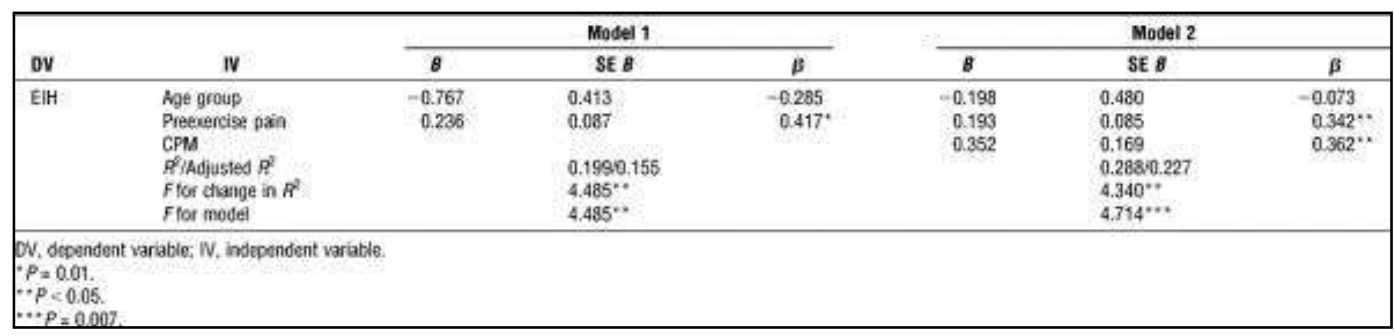

TABLE 2. Hierarchical regression analysis.

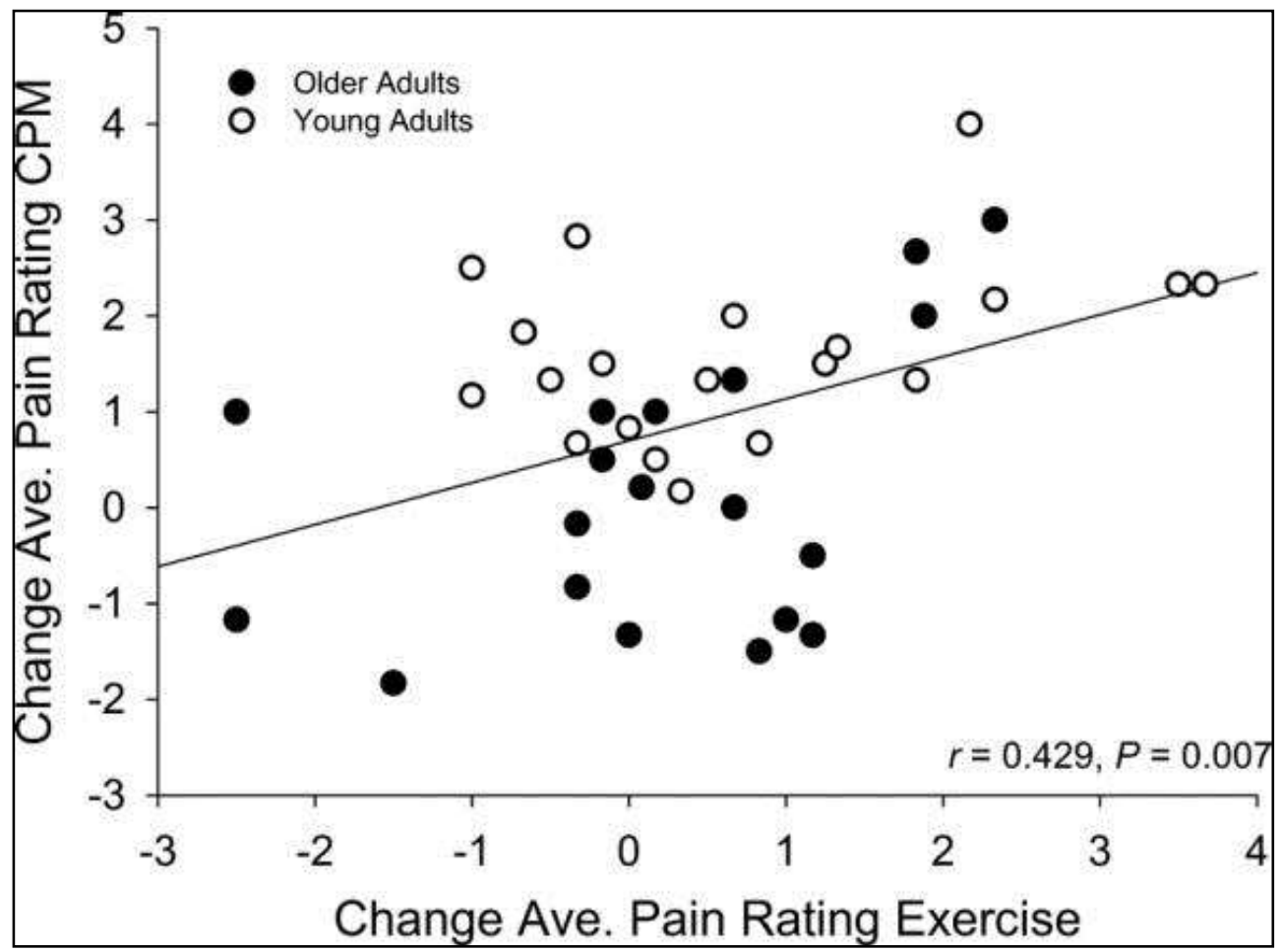

FIGURE 4. CPM-EIH relation. CPM efficiency is significantly correlated with the change in pressure pain ratings after exercise. Note that positive numbers represent a hypoalgesic response and negative numbers represent a hyperalgesic response.

Medicine \& Science in Sports \& Exercise, Vol 47, No. 1 (January 2015): pg. 176-184. DOI. This article is (C) American College of Sports Medicine and permission has been granted for this version to appear in e-Publications@Marquette. American College of Sports Medicine does not grant permission for this article to be further copied/distributed or hosted elsewhere without the express permission from American College of Sports Medicine. 